\title{
Effects of intensity on the categorical perception of stop consonants and isolated second formant transitions
}

\author{
ANN SYRDAL-LASKY \\ University of Minnesota, Minneapolis, Minnesota 55455
}

\begin{abstract}
Identification and discrimination of two-formant [bae-dae-gae] and [pae-tae-kae] synthetic speech stimuli and discrimination of corresponding isolated second formant transitions (chirps) were performed by six subjects. Stimuli were presented at several intensity levels such that the intensity of the F2 transition was equated between speech and nonspeech stimuli, or the overall intensity of the stimulus was equated. At higher intensity $(92 \mathrm{~dB}), \mathrm{b}-\mathrm{d}-\mathrm{g}$ and $\mathrm{p}-\mathrm{t}-\mathrm{k}$ identification and between-category discrimination performance declined and bilabial-alveolar phonetic boundaries shifted in location on the continuum towards the F2 steady-state frequency. Between-category discrimination improved from performance at $92 \mathrm{~dB}$ when $92-\mathrm{dB}$ speech stimuli were simultaneously masked by $60-\mathrm{dB}$ speech noise; alveolar-velar boundaries shifted to a higher frequency location in the 92-dB-plus-noise condition. Chirps were discriminated categorically when presented at $58 \mathrm{~dB}$, but discrimination peaks declined at higher intensities. Perceptual performance for chirps and p-t-k stimuli was very similar, and slightly inferior to performance for b-d-g stimuli, where simultaneous masking by $F 1$ resulted in a lower effective intensity of F2. The results were related to a suggested model involving pitch comparison and transitional quality perceptual strategies.
\end{abstract}

In categorical perception, a listener's ability to discriminate between two speech sounds is determined by his or her classification of the sounds into phonetic categories. When the two sounds are classified into different phonetic categories, they are highly discriminable, but when the two sounds are classified into the same phonetic category, even though the physical difference between the stimuli is the same as that of the between-category stimuli, they are discriminated at a very low level. The perception of various classes of phonetic categories has been found to be categorical: stop consonants differing in place of articulation (Liberman, Harris, Hoffman, \& Griffith, 1957; Mattingly, Liberman, Syrdal, \& Halwes, 1971), stop consonants differing in voicing (Abramson \& Lisker, 1967; Eimas \& Corbit, 1973; Lisker \& Abramson, 1967), liquids (Miyawaki,

This research was supported by grants to the Center for Research in Human Learning, University of Minnesota, Minneapolis, from the National Science Foundation (GB-17590), the National Institute of Child Health and Human Development (HD-00098), and the Graduate School of the University of Minnesota. I wish to thank Dr. James J. Jenkins for his help in planning the study. I am also grateful to Dr. Alvin M. Liberman and Dr. Franklin S. Cooper for making the facilities of Haskins Laboratories available to me for the production of stimuli used in the experiment. The author's current address is Phonetics Research Facility, 1629 Ellison Hall, University of California, Santa Barbara, California 93106. After July 1, 1978, the author's address will be University of Texas at Dallas, Callier Center for Communication Disorders, 1966 Inwood Road, Dallas, Texas 75235 .
Strange, Verbrugge, Liberman, Jenkins, \& Fujimura, 1975; McGovern \& Strange, 1977), fricatives (Fujisaki \& Kawashima, Note 1), and nasals (Miller \& Eimas, 1977). Such categorical perception contrasts with the continuous perception typical of simple nonspeech dimensions such as colors and pure tones (Miller, 1956; Pollack, 1952), in which discriminability is not limited by categorization. Several studies have examined discrimination of the acoustic cues used to differentiate synthetic speech sounds when isolated from their speech contexts: isolated second formants and second formant transitions used to cue place of articulation in stop consonants were studied by Mattingly et al. (1971), and isolated second formant transitions, by Popper (1972); isolated third formants used to differentiate liquids [r] from [1] were studied by McGovern and Strange (1977) and by Miyawaki et al. (1975). These studies found continuous, not categorical, perception of isolated acoustic cues, whereas the same cues embedded in a speech context resulted in categorical perception. Such perceptual differences between certain speech and closely related nonspeech sounds are cited frequently as evidence for the special nature of the speech mode of perception.

Recently, several studies examining categorical perception have challenged the claim that it is a phenomenon reflecting the unique nature of the perception of speech by humans. It has been found that a species other than humans-chinchillas-appears to exhibit categorical-like perception of stop consonants differing along the voicing continuum (Kuhl 
\& Miller, 1975). These results indicate that the categorical perception of at least some speech sounds need not be related to phonetic perceptual mechanisms unique to man, but that there must be an acoustic basis behind at least this abrupt perceptual discontinuity. The unique status of speech perception was also challenged by studies which found categorical perception by humans along nonlinguistic acoustic dimensions. Miller, Wier, Pastore, Kelly, and Dooling (1976), Pisoni (1977), and Stevens and Klatt (1974) argue that when two acoustically different components of a complex sound, whether speech or nonspeech, have onset times that differ by less than 20-25 msec, their onsets are perceived as simultaneous, whereas when their onset times are greater than 20-25 msec apart, the components are perceived as successive events. Another instance of categorical perception along a nonspeech continuum is represented by studies by Cutting and Rosner (1974) and Jusczyk, Rosner, Cutting, Foard, and Smith (1977). The perception of rise time-rapidity of stimulus onset-was found to be categorical in adults whether the stimuli presented were speech, sawtooth waves, or sine waves. Young infants also displayed categorical perception of sawtooth stimuli. The authors hypothesize that many of the "presumably unique features of human speech can be explained in terms of the acoustic properties of the sounds" (Jusczyk et al., 1977, p. 53).

Both auditory and phonetic bases for categorical perception have been proposed in an auditory memory framework by Fujisaki and Kawashima (Note 1, Note 2). They proposed that there is a shortterm auditory memory, which is essentially a neural "echo" of the physical stimulus and which decays rapidly, and a phonetic memory, a set of discrete features, that decays more slowly. In categorical perception of consonants, the high between-category discrimination is based on phonetic memory and the low within-category discrimination is based on acoustic memory, since phonetic memory does not distinguish between stimuli within the same phonetic category. Auditory memory for vowels, which are of greater duration and intensity than consonants, is presumed to be stronger than auditory memory for consonants, which are characteristically brief, transient, and of lower intensity. Work by Pisoni (1973, Note 3) supported this hypothesis. Between-category discrimination of both consonants and vowels was high and did not vary with the time delay intervals between discrimination pairs, presumably because the discrimination of both was based upon phonetic memory. Within-category discrimination, however, was consistently low for consonants (for which phonetic memory was uninformative and auditory memory was relatively weak), but high for vowels (for which phonetic memory was also uninformative but auditory memory was relatively strong), where discriminability decreased as the delay interval increased or other experimental procedures allowed the auditory memory to decay. Vowel perception has also been found to be more categorical when the vowels were degraded by being presented with noise (Lane, 1965) or when vowel duration and acoustic stability were reduced by embedding them in CVC syllables (Stevens, 1968; Sachs, Note 4). Either of these procedures can be viewed as reducing the usually strong acoustic memory for vowels and making them more similar to consonants in this respect. Thus, auditory and phonetic memories can reasonably be hypothesized to have a role in the phenomenon of categorical perception, but a very basic question remains: What is the acoustic basis for assignment of a speech sound to a phonetic category?

The present study examines several variables of interest in the perception of stops differing in place of articulation and their coresponding second formant transitions presented in isolation. These variables are related to possible auditory bases for the perception of speech. The question raised by Popper (1972) of whether the F1F3 context masks all but the phonemic differences in the F2 transition is addressed by employing three sets of stimuli: two formant voiced stops, two formant voiceless stops (in which the $F 2$ transition precedes the onset of $F 1$ and hence cannot be simultaneously masked by it), and isolated F2 transitions. The observation that low-energy transience is typical of categorically perceived speech is also explored by varying the intensity of the various stimuli presented and, in one condition, by degrading the speech stimuli by presenting them in noise. Little attention has been paid to the effects of intensity on speech or related nonspeech perception. A study by Dorman (1974) examined the discrimination of $0-9-\mathrm{dB}$ intensity differences on 50-msec F2 transitions in and out of syllable context, and on the first 50 msec of steady-state vowels. He found the intensity differences discriminable in vowels and isolated F2 transitions, but not in F2 transitions in syllable context. In further studies, Cutting and Dorman (1976) and Pastore, Ahroon, Puleo, Crimmins, Golowner, and Berger (1976) showed the differences in intensity discriminability to have an acoustic, rather than a phonetic, basis. Whether differences in intensity could affect other dimensions in stop consonant and related nonspeech perception has not been examined previously.

\section{METHOD}

\section{Subjects}

Subjects were six paid volunteers from a University of Minnesota introductory psychology course. Twelve students were initially interviewed for the experiment and given short speech identification pretests. Those eight who showed the better identification 
pretests and who were available during the scheduled testing periods were chosen as subjects. All were native English speakers. Individually administered audiometric examinations indicated that each subject had normal hearing; pure-tone air-conduction thresholds were measured at the frequencies of $250,500,1,000,2,000$, 4,000 , and $8,000 \mathrm{~Hz}$ in each ear. The subjects were tested in the experimental situation in groups of two. Of the eight subjects, two were dropped from the experiment because of very poor subsequent performance in identifying speech stimuli, missed appointments and other indications that they were unwilling to attend to the experimental tasks.

\section{Apparatus}

The following is a list of the apparatus used in all experimental conditions, in the order of their input-output connections: (1) a Crown Pro-800 tape recorder; (2) a Grason-Stadler Model 901B noise generator; (3) a decade attenuator, Hewlett Packard 350D attenuator set A1047; (4) a patch panel constructed at the University of Minnesota; (5) a Heathkit VTVM C1028 voltmeter and two TDH 39 earphones, to which the signal passed simultaneously. For measuring the sound pressure level at the ear, a Brüel and Kjaer sound-level meter and a Brüel and Kjaer NBS 9A coupler were employed.

\section{Stimulus Materials}

The stimuli were prepared on the parallel resonance synthesizer at Haskins Laboratories. The set of $16 \mathrm{~b}-\mathrm{d}-\mathrm{g}$ stimuli are shown in Figure 1a. Only first and second formants were synthesized, but were sufficient for the stimuli to be heard as [bae], [dae], or [gae]. The entire stimulus pattern was generated with a periodic buzz source of excitation (analogous to voicing in real speech). The first formant was constant for all stimuli. During the first $50 \mathrm{msec}$, the F1 transition rose in frequency from 100 to $692 \mathrm{~Hz}$; the last $250 \mathrm{msec}$ of $\mathrm{F} 1$ remained steady at $692 \mathrm{~Hz}$. The steady-state portion of the second formant was constant at $1,620 \mathrm{~Hz}$ for all stimuli. The $40-\mathrm{msec}$ second formant transition differentiated the 16 different stimuli. The initial point of the $16 \mathrm{~F} 2$ transitions ranged, in 15 approximately equal steps, from 1,075 to $2,234 \mathrm{~Hz}$, each ending at the F2 steady-state frequency. Each transition was - used in making up one stimulus. The stimuli were labeled consecutively from -7 to 8 ; these numbers referred to the number of steps below or above the steady-state frequency at which the F2 began.

The second set of speech stimuli, p-t-k, are illustrated in Figure $1 \mathrm{~b}$. These two-formant stimuli, heard as [pae], [tae], and [kae], were identical to the b-d-g set except for one feature. The initial $50 \mathrm{msec}$ of the first formant present in the b-d-g stimuli were deleted in synthesizing the p-t-k stimuli. This was sufficient for the perception of voiceless stops. For the $\mathrm{p}-\mathrm{t}-\mathrm{k}$ stimuli as well as the b-d-g, a buzz source of excitation was employed.

The nonspeech stimuli. or "chirps," are shown in Figure lc. They were simply the 40-msec second formant transitions identical to those used in the speech stimuli, but presented in isolation. That is, no first formant or second formant steady-state portions were included. The subjects did not report that chirps sounded speech-like when asked to describe them during the experiment; they typically were reported as sounding like birds' chirps.

Four audio tapes were prepared for each of the two sets of speech stimuli-three for a discrimination task and one for an identification task. Three discrimination tapes were made for the chirp stimuli. A tape contained only stimuli within one set.

For the discrimination task, stimuli were presented in oddity triads. Two identical stimuli were presented along with one different stimulus. These three stimuli were presented sequentially with a 1 -sec interstimulus interval. The "odd" stimulus could occur in any of the three positions in the triad. A given stimulus was compared in a triad only with another stimulus two steps, approximately $155 \mathrm{~Hz}$, different from it. Since there were 16 stimuli in a set, 14 such comparisons were possible. Each discrimination tape
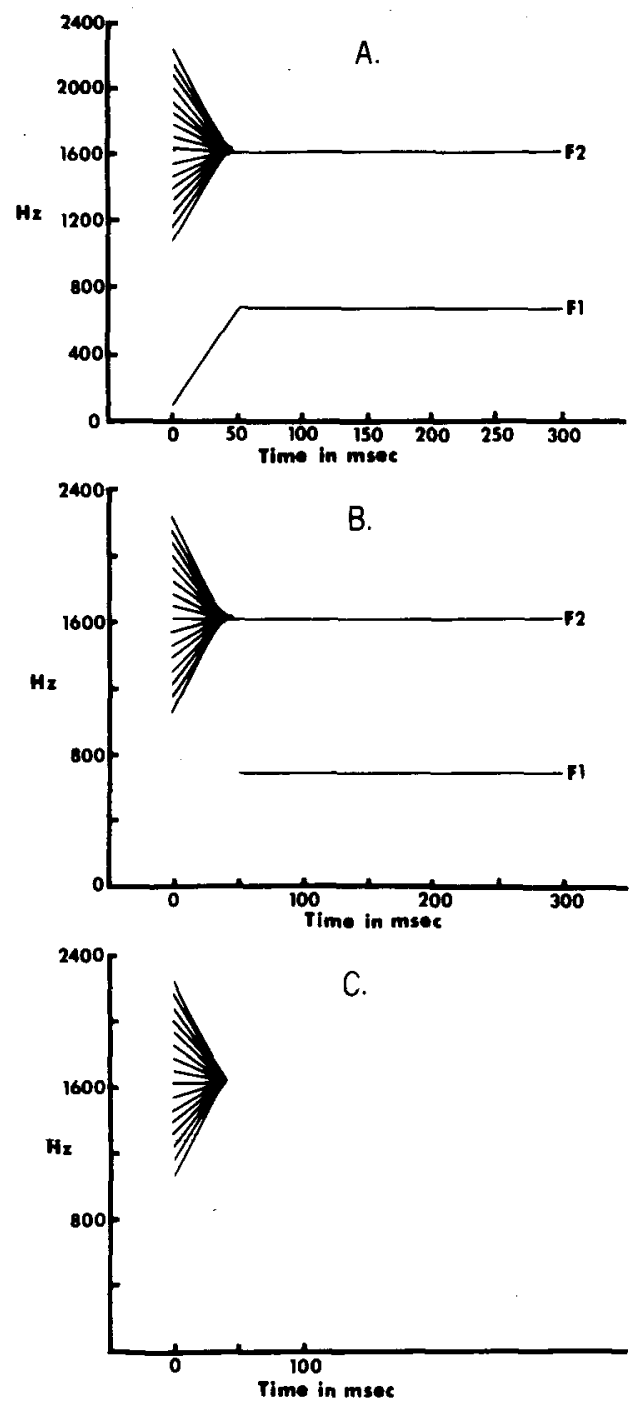

Figure 1. (A) Voiced stop stimuli [bae-dae-gae]. (B) Voiceless stop stimuli [pae-tae-kae]. (C) Chirp stimuli (isolated $\mathbf{F}$ transitions).

was arranged in six blocks of 14 triads each; each of the possible 14 comparisons was made once within each block. For each possible comparison, there were three possible positions within the triad for the odd stimulus. In addition, there were two possibilities between the two stimuli being compared as to which would be the odd stimulus presented only once in the triad. Thus, for a comparison between two particular stimuli, there were six different triads which could be constructed. Each of these six triads was presented within a discrimination tape. Within the above constraints, stimulus triads were presented in random order. The same ordering was employed for each of the three stimulus sets. Within a block, there was a 3-sec interval between triads. There was a 5 -sec interval between the last triad in one block and the first triad in the next block. The duration of an entire discrimination tape was about $9 \mathrm{~min}$. Each of the three discrimination tapes prepared for each stimulus set had a different random ordering of stimuli.

One identification tape was also prepared for each of the two sets of speech stimuli-b-d-g and p-t-k. Stimuli in these tapes were presented individually. There were 10 blocks of 16 stimuli; each stimulus was presented once within a block. Stimuli within a block were presented in random order. The same ordering was 
employed for both stimulus sets. The interstimulus interval was $3 \mathrm{sec}$ within a block and $6 \mathrm{sec}$ between blocks. The duration of an identification tape was approximately $11 \mathrm{~min}$.

\section{Procedure}

The subjects were tested in four groups of iwo subjects each for seven 1-h sessions.

For the discrimination tasks, the subjects were instructed to listen carefully to all three sounds in a triad. They were told that there were always two identical sounds and one different sound in each triad. It was their job to determine which - the first, second, or third-sound was the different one. They were to indicate their choices by marking the appropriate box (one, two, or three) on a mimeographed answer sheet. The subjects were required to respond to every triad.

In the identification test, the subject's task was to label each speech sound heard on the identification tape. He was given an answer sheet with a numbered blank for each stimulus, and was instructed to write the symbol of the consonant sound he heard--b, d, or $\mathrm{g}$, in one case, or $\mathrm{p}, \mathrm{t}$, or $\mathrm{k}$, in the other-in the appropriate space.

Discrimination and identification stimulus tapes were presented at the following intensity levels, which were attained solely by use of a decade attenuator, so that no distortion of the stimuli due to operating characteristics of the tape recorder was introduced: (1) b-d-g at $75 \mathrm{~dB} \mathrm{SPL}, 92 \mathrm{~dB}$ SPL, and $92 \mathrm{~dB}$ SPL with 60-dB SPL speech noise; (2) p-t-k at $75 \mathrm{~dB}$ SPL, $92 \mathrm{~dB}$ SPL, and $92 \mathrm{~dB}$ SPL with 60-dB SPL speech noise; and (3) chirps at 58,75, and $92 \mathrm{~dB}$ SPL. These sound pressure levels were chosen because of several considerations. First, the difference in intensity between the speech stimuli and the chirps was measured to be $17 \mathrm{~dB}$ when both were played at the same gain setting (all stimuli were recorded at the same level, and a constant calibration tone preceded each tape so that equality of sound levels could be easily verified using a voltmeter). Since it was desirable to present the chirps at the same intensity as the speech with which they were compared, as well as at the intensity of $\mathbf{F} 2$ transitions actually present in the speech conditions, it was decided to present the lower and higher intensity levels of speech at a difference of $17 \mathrm{~dB}$. Studies of the intelligibility of real speech (Miller, 1951) found that intelligibility was essentially optimal at both intensity levels (though slightly higher at $92 \mathrm{~dB}$ than at $75 \mathrm{~dB}$ ).

During the first four experimental sessions, discrimination tape randomizations 1 and 2 were administered. During the last $15 \mathrm{~min}$ of the fourth testing session and the entire fifth session, subjects were administered identification tests. The third randomization of the discrimination task for the three sets of stimuli was presented during the last two experimental sessions. Orders of presentation of stimulus sets and intensity levels were counterbalanced between subjects and within sessions.

The data collected from each subject in the experiment were 18 discrimination judgments for each of 14 comparison pairs of each of three stimulus sets at each of three intensity levels, and 10 identifications of each of 16 stimuli of each of two speech stimulus sets at each of three intensity levels.

\section{RESULTS AND DISCUSSION}

The following discussion of results will deal with three experimental conditions. The discussion will progress in a fashion designed to facilitate explanation, and does not relate to the order in which the experimental conditions were presented.

\section{Experiment 1: b-d-g}

The b-d-g condition was intended to replicate the phenomenon of categorical perception and to investi- gate what changes may occur in identification and discrimination of these stimulis over different intensity levels.

Figure 2 illustrates the effect of intensity changes in the perception of $b-d-g$ stimuli showing pooled data from all six subjects. Figure $2 a$ shows the $b-d-g$ identification functions at $75 \mathrm{~dB}, 92 \mathrm{~dB}$, and $92 \mathrm{~dB}$ plus noise. Figure $2 \mathrm{~b}$ shows the $\mathrm{b}-\mathrm{d}-\mathrm{g}$ pooled discrimination functions for the same three intensity levels. The numbers along the abscissa of each graph refer to the stimulus number. Each stimulus, from -7 to 8 , is included in the identification graph, since all 16 stimuli were presented individually for identification. The stimulus numbers on the discrimination graph, however, refer to the stimulus between the two compared on a triad. For example, -6 refers to a comparison between -7 and -5 . Since those stimuli compared in a triad were always two steps apart, numbers representing discrimination pairs on the discrimination graph range from -6 to 7 . The ordinate of the identification graph indicates what percent of the time, based on 10 identifications of each stimulus per subject, a particular stimulus was identified in a certain way. The ordinate of the discrimination graph refers to the percent of correct discriminations that were made out of 18 possible per subject (108 total) for each comparison. One-third of the discriminations would be expected to be correct by chance, and this random level of responding is indicated on the graph.

The three identification functions along the b-d-g continuum differ in several respects. The left half of Table 1 lists the individual and mean boundary loci and the width of the middle $d$ category (the difference between the two loci) for each intensity level. The 75-dB b-d locus was at a significantly lower frequency than the $92-\mathrm{dB}$ and $92-\mathrm{dB}$-plus-noise b-d loci by sign tests (each $\mathrm{p}=.032$, two-tailed). The 92-dB-plus-noise d-g locus was significantly higher in frequency than the 75- and 92-dB d-g loci $(\mathrm{p}=.032$, sign tests, two-tailed). The width of the $d$ category was significantly greater at 75 than at $92 \mathrm{~dB}(\mathrm{p}=$ .032 , sign test, two-tailed). The $92-\mathrm{dB}$ and $92-\mathrm{dB}$ plus-noise b-d loci did not differ statistically, nor did the 75 and $92-\mathrm{dB}$ d-g loci. It is apparent, by inspection of Figure $2 a$, that the steepness of the slopes of the 75-dB identification functions were greater than those at $92 \mathrm{~dB}$; the 92 -dB-plus-noise slopes were also steeper than those at $92 \mathrm{~dB}$, but not so steep at $75 \mathrm{~dB}$. The consistency of d identification judgments, as indicated by height of the d category, was also greatest at $75 \mathrm{~dB}$ and lowest at $92 \mathrm{~dB}$.

The extent to which stimuli were perceived categorically was examined by comparing each subject's expected discrimination function predicted from his identification function to his observed discrimination function for the same stimulus condition and inten- 

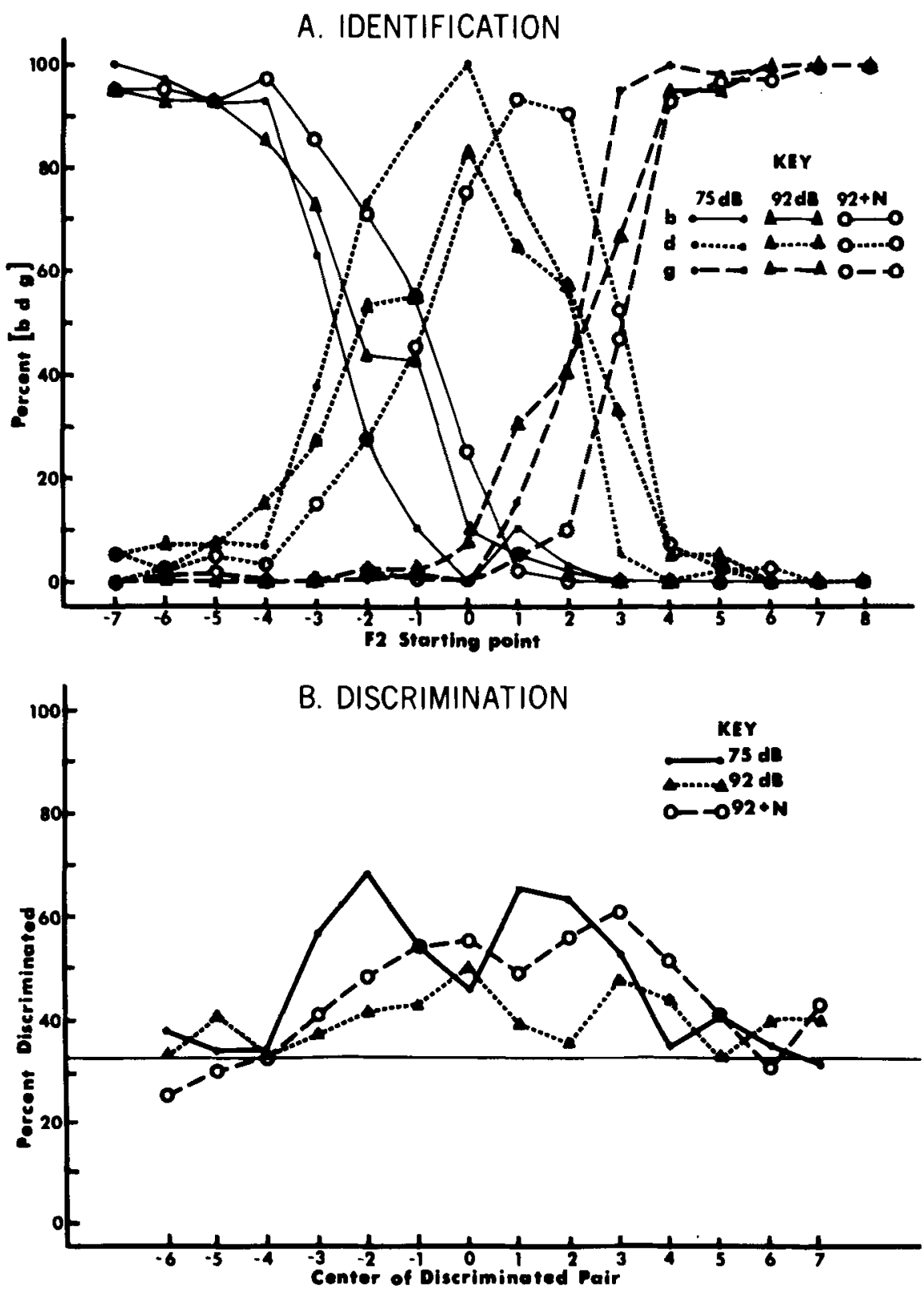

Figure 2. (A) Pooled identification functions for voiced stops at $75 \mathrm{~dB}, 92 \mathrm{~dB}$, and $92 \mathrm{~dB}$ plus noise. (B) Pooled discrimination functions for voiced stops at $75 \mathrm{~dB}, 92 \mathrm{~dB}$, and $92 \mathrm{~dB}$ plus noise.

sity level. A formula was calculated to predict discrimination functions for an oddity discrimination paradigm like that used in this study from identification functions when there were three possible phonetic categories that could be perceived. The resulting formula is:

$$
\begin{aligned}
\mathbf{P}_{\text {correct }}= & \left\{1+2\left[\left(\mathbf{P}_{1 \mathrm{~b}}-\mathbf{P}_{2 \mathrm{~b}}\right)^{2}+\left(\mathbf{P}_{1 \mathrm{~g}}-\mathbf{P}_{2 \mathrm{~g}}\right)^{2}\right.\right. \\
& \left.\left.+\left(\mathbf{P}_{1 \mathrm{~b}}-\mathbf{P}_{2 \mathrm{~b}}\right)\left(\mathbf{P}_{1 \mathrm{~g}}-\mathbf{P}_{2 \mathrm{~g}}\right)\right]\right\} / 3,
\end{aligned}
$$

where $\mathrm{P}_{\text {correct }}$ is the proportion correctly discriminated for a specific comparison in an oddity test, and $P_{n x}$ is the proportion of identification trials where stimulus $n$ was identified as category $x$. In the above formula, Stimuli 1 and 2 refer to the two stimuli compared in a discrimination triad, and categories $b$ and $g$ were used to refer to the two "end" categories on the continuum which were separated by a middle category. Each subject's predicted discrimination function was computed for each identification test condition. In each condition, each subject's predicted and obtained discriminations were compared for each discrimination comparison pair by a chi-square test ( $\mathrm{df}=1$ ) according to the formula used by Pisoni (1971): 
Table 1

Individual and Mean b-d-g and p-t-k Boundary Loci and Middle Category Width for Each Intensity Level

\begin{tabular}{|c|c|c|c|c|c|c|c|c|}
\hline \multirow[b]{2}{*}{ Subject } & \multicolumn{4}{|c|}{$b-d-g$} & \multicolumn{4}{|c|}{$p-t-k$} \\
\hline & & 75 & 92 & $92 \& N$ & & 75 & 92 & $92 \& N$ \\
\hline 1 & $\begin{array}{l}b-d \\
d-g \\
d \text { width }\end{array}$ & $\begin{array}{r}-2.25 \\
2.50 \\
4.75\end{array}$ & $\begin{array}{r}-.43 \\
2.00 \\
2.43\end{array}$ & $\begin{array}{r}-1.25 \\
3.17 \\
4.42\end{array}$ & $\begin{array}{l}p-t \\
t-k \\
t \text { width }\end{array}$ & $\begin{array}{r}-1.00 \\
3.33 \\
4.33\end{array}$ & $\begin{array}{r}-.20 \\
2.16 \\
2.36\end{array}$ & $\begin{array}{r}1.90 \\
2.40 \\
.50\end{array}$ \\
\hline 2 & $\begin{array}{l}\text { b-d } \\
d-g \\
\text { d width }\end{array}$ & $\begin{array}{r}-2.00 \\
1.75 \\
3.75\end{array}$ & $\begin{array}{r}-.80 \\
2.33 \\
3.13\end{array}$ & $\begin{array}{r}-.56 \\
2.80 \\
3.36\end{array}$ & $\begin{array}{l}\text { p-t } \\
\text { t-k } \\
\text { t width }\end{array}$ & $\begin{array}{r}-.25 \\
.70 \\
.95\end{array}$ & $\begin{array}{r}-.50 \\
2.38 \\
2.88\end{array}$ & $\begin{array}{r}.67 \\
2.64 \\
1.97\end{array}$ \\
\hline 3 & $\begin{array}{l}\mathrm{b}-\mathrm{d} \\
\mathrm{d}-\mathrm{g} \\
\mathrm{d} \text { width }\end{array}$ & $\begin{array}{r}-3.00 \\
1.38 \\
4.38\end{array}$ & $\begin{array}{r}-2.59^{*} \\
.33 \\
2.92\end{array}$ & $\begin{array}{r}.29 \\
2.71 \\
2.42\end{array}$ & $\begin{array}{l}p-t \\
t-k \\
t \text { width }\end{array}$ & $\begin{array}{r}-1.45 \\
2.10 \\
3.55\end{array}$ & $\begin{array}{r}-.25 \\
2.00 \\
2.25\end{array}$ & $\begin{array}{l}0 \\
2.20 \\
2.20\end{array}$ \\
\hline 4 & $\begin{array}{l}\mathrm{b}-\mathrm{d} \\
\mathrm{d}-\mathrm{g} \\
\mathrm{d} \text { width }\end{array}$ & $\begin{array}{r}-3.00 \\
2.63 \\
5.63\end{array}$ & $\begin{array}{r}-2.00 \\
3.25 \\
5.25\end{array}$ & $\begin{array}{r}-1.00 \\
3.43 \\
4.43\end{array}$ & $\begin{array}{l}p-t \\
t-k \\
t \text { width }\end{array}$ & $\begin{array}{r}-.50 \\
4.00 \\
4.50\end{array}$ & $\begin{array}{c}-1.67 \\
4.50^{*} \\
6.17\end{array}$ & $\begin{array}{l}0 \\
3.67 \\
3.67\end{array}$ \\
\hline 5 & $\begin{array}{l}b-d \\
d-g \\
d \text { width }\end{array}$ & $\begin{array}{r}-3.67 \\
2.28 \\
5.95\end{array}$ & $\begin{array}{r}-2.59 \\
2.25 \\
4.84\end{array}$ & $\begin{array}{c}-.96^{*} \\
2.60 \\
3.56\end{array}$ & $\begin{array}{l}\text { p-t } \\
\text { t-k } \\
\text { t width }\end{array}$ & $\begin{array}{r}-1.50 \\
2.40 \\
3.90\end{array}$ & $\begin{array}{r}-.33 \\
2.35 \\
2.68\end{array}$ & $\begin{array}{c}-.67 \\
2.94 * \\
3.61\end{array}$ \\
\hline 6 & $\begin{array}{l}b-d \\
d-g \\
d \text { width }\end{array}$ & $\begin{array}{r}-2.67 \\
2.00 \\
4.67\end{array}$ & $\begin{array}{c}-1.74 \\
2.00^{*} \\
3.74\end{array}$ & $\begin{array}{r}-1.86 \\
3.38 \\
5.24\end{array}$ & $\begin{array}{l}p-t \\
t-k \\
t \text { width }\end{array}$ & $\begin{array}{r}-1.50 \\
2.38 \\
3.88\end{array}$ & $\begin{array}{r}-1.33 \\
2.00 \\
3.33\end{array}$ & $\begin{array}{r}-1.38 \\
2.33 \\
3.71\end{array}$ \\
\hline Mean & $\begin{array}{l}b-d \\
d-g \\
d \text { width }\end{array}$ & $\begin{array}{r}-2.77 \\
2.09 \\
4.86\end{array}$ & $\begin{array}{r}-1.69 \\
2.03 \\
3.72\end{array}$ & $\begin{array}{r}-.89 \\
3.02 \\
3.91\end{array}$ & $\begin{array}{l}p-t \\
t-k \\
t \text { width }\end{array}$ & $\begin{array}{r}-1.03 \\
2.49 \\
3.52\end{array}$ & $\begin{array}{r}-.71 \\
2.57 \\
3.28\end{array}$ & $\begin{array}{r}.09 \\
2.70 \\
2.61\end{array}$ \\
\hline
\end{tabular}

*Mean of two boundary crossovers.

$$
\chi^{2}=N \frac{\left(p_{o}-p_{e}\right)^{2}}{p_{e} \cdot \frac{q_{e}}{}}
$$

The Pearson chi-square statistic for goodness of fit was employed to compare predicted and obtained discrimination functions for each condition (Pisoni, $1971,1977)$. The results of the separate comparisons for each condition for each subject is presented in Table 2; the upper three rows represent the three intensity levels at which b-d-g stimuli were presented. The poor match between $75-\mathrm{dB}$ b-d-g predicted and obtained discrimination functions was the result of the fact that some $d-g$ peaks in discrimination functions occurred in different locations on the continuum than predicted. Identification functions indicate that a discrimination peak would be expected at pair 2 or pair 3, but the peak's highest point was located instead at pair 1. Furthermore, since $75-\mathrm{dB}$ b-d-g stimuli were so abruptly divided into discrete categories, the predicted proportion discriminated correctly was frequently about 1.0 at between-category boundaries; consequently, if the observed proportion correct was more than very slightly discrepant from 1.0 , the resulting chi square was very large. The latter explanation also accounted for the two extremely high chi squares found at $92 \mathrm{~dB}$ plus noise.

Despite the frequently poor fits between predicted and observed discrimination functions, there were consistencies between b-d-g identification and dis-

Table 2

Comparison of Predicted and Obtained Discrimination Functions

\begin{tabular}{|c|c|c|c|c|c|c|c|}
\hline \multirow[b]{2}{*}{ Predicted/Obtained } & \multirow[b]{2}{*}{$\mathrm{df}$} & \multicolumn{6}{|c|}{ Subject } \\
\hline & & 1 & 2 & 3 & 4 & 5 & 6 \\
\hline $75 \mathrm{~dB}$ bdg/75 dB bdg & 13 & $63.74^{*}$ & 18.48 & $159.59^{*}$ & $181.97 *$ & $40.28 *$ & $34.98^{*}$ \\
\hline $92 \mathrm{~dB}$ bdg/92dB bdg & 13 & 15.43 & $56.98^{*}$ & 18.52 & 28.24 & 18.46 & 8.43 \\
\hline $92 \mathrm{~dB} \& \mathrm{~N}$ bdg/92 dB \& $\mathrm{N}$ bdg & 13 & 27.74 & $219.50^{*}$ & $899.97^{*}$ & 25.00 & 10.65 & 26.13 \\
\hline $75 \mathrm{~dB}$ ptk/75 dB ptk & 13 & 10.23 & 26.05 & $43.07^{*}$ & 30.23 & 12.47 & 18.39 \\
\hline $92 \mathrm{~dB}$ ptk $/ 92 \mathrm{~dB}$ ptk & 13 & 14.19 & $51.63^{*}$ & $35.08^{*}$ & 28.40 & 15.02 & 17.65 \\
\hline $92 \mathrm{~dB} \& \mathrm{~N}$ ptk/92 dB \& N ptk & 13 & 17.00 & 19.22 & 24.90 & $52.90^{*}$ & 11.40 & $51.02 *$ \\
\hline $75 \mathrm{~dB}$ bdg $/ 58 \mathrm{~dB}$ chirps & 12 & $35.84^{*}$ & $223.23^{*}$ & $686.33^{*}$ & $611.13^{*}$ & 20.95 & $44.47^{*}$ \\
\hline $92 \mathrm{~dB}$ bdg $/ 75 \mathrm{~dB}$ chirps & 12 & $45.59 *$ & 31.33 & 21.64 & 30.67 & 21.04 & 20.98 \\
\hline $75 \mathrm{~dB} p t k / 58 \mathrm{~dB}$ chirps & 12 & 28.68 & 31.22 & 27.81 & $49.38^{*}$ & 11.65 & $\$ 3.00$ \\
\hline $92 \mathrm{~dB}$ ptk $/ 75 \mathrm{~dB}$ chirps & 12 & 26.80 & 30.67 & 27.14 & 20.35 & 17.84 & 12.58 \\
\hline
\end{tabular}


crimination functions. Between-category discrimination, revealed by the height of discrimination peaks, was superior at $75 \mathrm{~dB}$, less so at $92 \mathrm{~dB}$ plus noise, and poorest at $92 \mathrm{~dB}$; these same rankings were seen in steepness of identification function slopes. An Intensity Level by Discrimination Pair repeated measures analysis of variance, with both intensity and discrimination pairs as repeated factors, was performed on the discrimination data of all six subjects. The number of correct discriminations made by each subject for each b-d-g discrimination pair was compared at the three intensity levels. There were significant differences between the overall discrimination of different pairs $(F=7.01, \mathrm{df}=13 / 195, \mathrm{p}<.001)$, and peaks occurred in significantly different locations at different intensities $(F=2.20$, df $=26 / 195$, $\mathrm{p}<.001$ ). Both 75-dB peaks were at lower frequencies than 92-dB and 92-dB-plus-noise peaks. Tukey test specific contrasts (with $\mathrm{p}<.05$ ) indicated that at $75 \mathrm{~dB}$ there were two discrimination peaks, each consisting of three stimulus pairs: $-3,-2-1$ and $1,2,3$. Both peaks were significantly higher than the remaining troughs to the left, right, and between the peaks. At $92 \mathrm{~dB}$ there were two peaks, each consisting of only one discrimination pair: 0 and 3 . The $92-\mathrm{dB}$ peaks were significantly greater than only three other points in the discrimination function, and were not significantly higher than the trough between them. The discrimination peaks at $92 \mathrm{~dB}$ plus noise-consisting of discrimination pairs $-1,0$ and $2,3,4$-were significantly larger than discrimination troughs at the lower and higher ends of the frequency continuum, but not significantly larger than the trough between them. The highest points of both 75-dB discrimination peaks were significantly higher than the two 92-dB peaks, but not significantly higher than the 92-dBplus-noise peaks.

Changes in intensity produced two main effects in the perception of b-d-g stimuli: identification boundaries and discrimination peaks changed locations along the frequency continuum, and discrimination peak heights decreased from the highest level at $75 \mathrm{~dB}$ to $92 \mathrm{~dB}$ plus noise to the lowest level at $92 \mathrm{~dB}$. The latter results seem the opposite of what one might expect; as stimulus intensity was increased from 75 to $92 \mathrm{~dB}$, between-category discrimination was poorer, rather than better, and when masking noise was added to 92-dB stimuli, between-category discrimination improved, rather than deteriorated. The reduced between-category discrimination performance at $92 \mathrm{~dB}$ relative to $75 \mathrm{~dB}$ may be related to the simultaneous presence of a lower frequency first formant which may have masked the F2 transition (Rand, 1974, found masking effects of about $20 \mathrm{~dB}$ on F2F3 produced by $\mathrm{F} 1$ in similar stimuli) more at higher intensities (Flanagan \& Saslow, 1958), resulting in poorer discriminability at higher inten- sities than at lower intensities. The improvement of between-category discrimination when noise was added to $92-\mathrm{dB}$ stimuli would seem to argue against this hypothesis, however. Experiment 2 was designed to test the hypothesis further.

\section{Experiment 2: p-t-k}

The series of voiceless two-formant stops Ipae, tae, kae] presented in Experiment 2 lacked a first formant during the first $50 \mathrm{msec}$ of the stimulus. Thus, the 40-msec F2 transition could not have been simultaneously masked by any other formant during its presentation. To preserve the comparability of F2 transitions in voiced and voiceless stop contexts, the transition was synthesized with a buzz source in both conditions (normally, the F2 transition of an aspirated stop would be synthesized with a hiss source). P-t-k stimuli were also presented at $75 \mathrm{~dB}, 92 \mathrm{~dB}$, and $92 \mathrm{~dB}$ plus $60-\mathrm{dB}$ speech noise to compare the perceptual results of intensity level with those observed with b-d-g stimuli. If the poorer perceptual performance for b-d-g stimuli at $92 \mathrm{~dB}$ were caused by increased simultaneous masking of the $\mathrm{F} 2$ transition by $\mathrm{F} 1$ at the higher intensity, the perceptual differences observed between 75 - and $92-\mathrm{dB}$ b-d-g stimuli would not be expected to occur between 75- and 92-dB p-t-k stimuli. It has also been suggested (Popper, 1972) that the acoustic basis of discriminability between voiced stops may involve simultaneous masking effects of the F2 transition by other formants present in the speech stimulus. Discrimination of voiceless two-formant stops is relevant to this hypothesis also, since there were no simultaneously occurring components of the stimulus along with the F2 transition.

Figure $3 a$ displays pooled $p-t-k$ identification functions for $75 \mathrm{~dB}, 92 \mathrm{~dB}$, and $92 \mathrm{~dB}$ plus noise. The right half of Table 1 lists the individual and mean $p$-t-k boundary loci and $t$ category widths for each intensity level. The 75-dB p-t locus was significantly lower in frequency than the 92-dB-plus-noise $p-t$ locus by a sign test ( $p=.032$, two-tailed). There were several significant differences (by sign test, with $\mathrm{p}=.032$, two-tailed) between the b-d-g and $\mathrm{p}-\mathrm{t}-\mathrm{k}$ boundary loci and middle category widths. The 75-dB b-d boundary was located at a significantly lower frequency than the p-t boundary at all three intensities; the 92-dB b-d boundary was significantly lower in frequency than the $92-\mathrm{dB}$ and 92 -dB-plusnoise p-t boundaries; the $92-\mathrm{dB} \mathrm{d}-\mathrm{g}$ boundary occurred at a significantly lower frequency than the 92-dBplus-noise $\mathrm{t}-\mathrm{k}$ boundary; the $75-\mathrm{dB} \mathrm{d}$ category was significantly wider than the $75-\mathrm{dB}$ and the 92-dBplus-noise $t$ categories; the $92-\mathrm{dB} d$ category was significantly wider than the 92-dB-plus-noise t category. When results from the two speech conditions were combined in order to consider what changes in place of articulation feature boundaries and alveolar cate- 

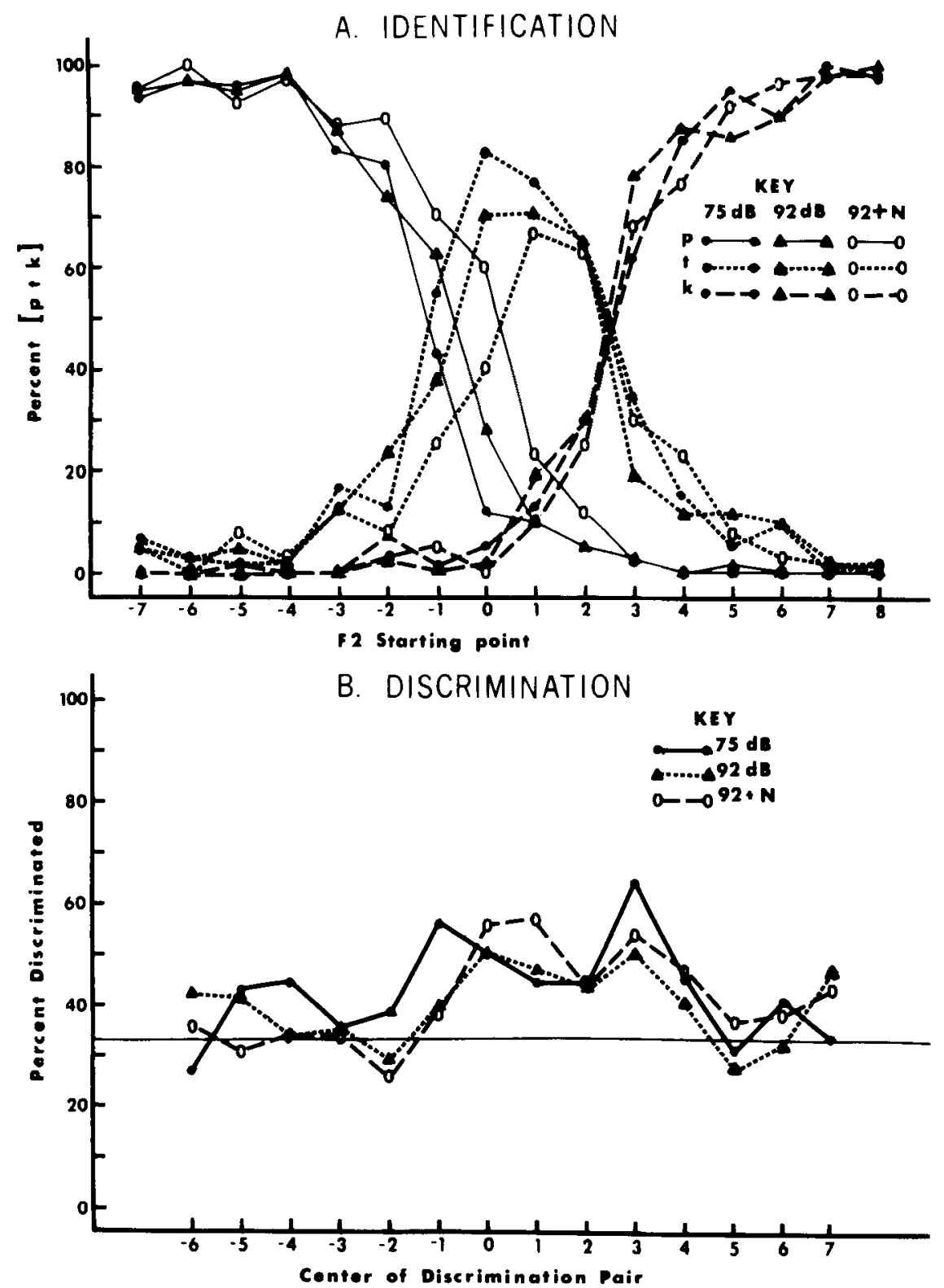

Figure 3. (A) Pooled identification functions for voiceless stops at $75 \mathrm{~dB}, 92 \mathrm{~dB}$, and $92 \mathrm{~dB}$ plus noise. (B) Pooled discrimination functions for voiceless stops at $75 \mathrm{~dB}, 92 \mathrm{~dB}$, and $92 \mathrm{~dB}$ plus noise.

gory widths occurred over the three different intensities, the following effects were significant by sign tests: the bilabial-alveolar boundary was located at a lower frequency at $75 \mathrm{~dB}$ than at both $92 \mathrm{~dB}(\mathrm{p}=$ .038 , two-tailed) and $92 \mathrm{~dB}$ plus noise ( $<<.006$, two-tailed); the alveolar-velar boundary was located at a higher frequency at $92 \mathrm{~dB}$ plus noise than at $92 \mathrm{~dB}(\mathrm{p}=.006$, two-tailed). The alveolar category was wider at $75 \mathrm{~dB}$ than at both $92 \mathrm{~dB}(\mathrm{p}=.038)$ and $92 \mathrm{~dB}$ plus noise $(\mathrm{p}=.038)$. The $\mathrm{t}$ category over all intensity levels was significantly narrower than the $d$ category, by sign test $(p=.002$, twotailed).

Figure $3 \mathrm{~b}$ shows the $\mathrm{p}-\mathrm{t}-\mathrm{k}$ discrimination functions at $75 \mathrm{~dB}, 92 \mathrm{~dB}$, and $92 \mathrm{~dB}$ plus noise. The middle three rows of Table 2 show individual chi-square values for predicted and obtained p-t-k discrimination functions at the three intensities. Predicted and obtained discrimination functions were relatively more comparable than b-d-g functions, and chi-square values were in the same range as those of stop consonants reported by Pisoni (1971). An Intensity Level by Discrimination Pair repeated measures analysis of variance like that employed in the $b-d-g$ condition indicated that there were significant differences in the overall discrimination of different pairs $(F=7.70$, $\mathrm{df}=13 / 195, \mathrm{p}<.001)$ and that discrimination peaks varied significantly in location over different intensities $(\mathrm{F}=1.60$, df $=26 / 195, \mathrm{p}<.039)$. Tukey-test specific contrasts (with $\mathrm{p}<.05$ ) indicated that there 
were two significant discrimination peaks at $75 \mathrm{~dB}$, $92 \mathrm{~dB}$, and $92 \mathrm{~dB}$ plus noise. The 75-dB peaks-consisting of pairs $-1,0$ and 3,4-were significantly larger than the trough areas above, below, and between them. The 92-dB peaks-consisting of pairs 0,1 and $3-$ were significantly higher than portions of the two end troughs of the discrimination function, but not higher than the trough between them. The 92-dB-plus-noise $\mathrm{p}-\mathrm{t}-\mathrm{k}$ discrimination peakspairs 0,1 and 3,4-were significantly larger than the troughs at the upper and lower ends of the function, and the p-t peak was larger than the trough between the peaks. The t-k peak was significantly higher at $75 \mathrm{~dB}$ than at $92 \mathrm{~dB}$.

The fact that between-category discrimination was better at $75 \mathrm{~dB}$ than at $92 \mathrm{~dB}$ for p-t-k stimuli as well as for b-d-g stimuli indicates that poorer performance was not caused by increased simultaneous masking of the $\mathrm{F} 2$ transition by $\mathrm{F} 1$ at the higher intensity level. The improved performance when noise was added to 92-dB stimuli reinforces this conclusion. There remains a possibility that increased backward masking at higher intensities may have been responsible for the poorer between-category discrimination at $92 \mathrm{~dB}$ than at $75 \mathrm{~dB}$. Although there were no appreciable backward masking effects observed with acoustically simple sounds at a 50-msec interval (Wilson \& Carhart, 1971; Elliott, 1962) and StuddertKennedy, Shankweiler, and Schulman (1970) found no backward masking of one syllable on another at a stimulus onset asynchrony of $50 \mathrm{msec}$, Massaro $(1973,1975)$ has claimed that recognition backward masking is effective over longer intervals than detection backward masking, including intervals well over $50 \mathrm{msec}$. Since between-category discrimination was better at $92 \mathrm{~dB}$ plus noise than at $92 \mathrm{~dB}$, however, it seems unlikely that backward masking could account for the results.

The fact that the p-t-k stimuli were perceived categorically indicates that the possible masking effect of F1 on the F2 transition cannot account for categorical perception, as Popper (1972) suggested it might: Since the possibility of simultaneous masking of $F 2$ transition by $F 1$ was eliminated by the absence of $F 1$ during $F 2$ transition in $\mathrm{p}$-t-k stimuli, and since a backward masking explanation also seems unlikely, it would appear that the acoustic cues that form the basis of the phonetic classification of stop consonants along the dimension of place of articulation are, indeed, the F2 transitions themselves. Another argument leads to the same conclusion; the improvement in between-category discrimination when noise was added to $92 \mathrm{~dB}$ stimuli must be the result of changes in the auditory basis underlying phonetic memory, and not phonetic memory itself, since the phonetic categories perceived were the same.

Although the pattern of changes related to intensity levels was quite similar for voiced and voiceless stim- uli, there were differences between them. At every intensity level, b-d identification boundary loci were consistently at lower frequencies than p-t loci at corresponding intensities. These differences were reflected in locations of discrimination peaks as well. By comparison of the $b-d-g$ and $p-t-k$ discrimination functions, it is apparent that 75-dB b-d-g peaks were somewhat higher than $75-\mathrm{dB}$ p-t-k peaks, although the peak heights at $92 \mathrm{~dB}$ and $92 \mathrm{~dB}$ plus noise were similar. Since F2 transitions in p-t-k stimuli were unmasked, they were presumably perceived at a higher effective intensity than the F2 transitions partially masked by $F 1$ in the b-d-g stimuli. The difference in effective perceptual intensity between b-d-g and p-t-k stimuli may have been responsible in some way for the perceptual performance differences observed between b-d-g and p-t-k stimuli, which follow much the same pattern as that observed over different intensities within voiced and voiceless sets.

Experiments 1 and 2 suggested, then, that there is an auditory quality present in the $F 2$ transition responsible for categorical perception along the continuum of place of articulation of stop consonants, and that this auditory quality is more salient at lower effective intensities than at higher effective intensities. Experiment 3 examined the effect of intensity on the discrimination of isolated $\mathrm{F} 2$ transitions.

\section{Experiment 3: Chirp Discrimination}

Chirps were presented at three intensity levels58,75 , and $92 \mathrm{~dB}$. The $58-\mathrm{dB}$ level corresponded to the level of the $F 2$ transition alone when the entire speech stimulus was presented at $75 \mathrm{~dB}$. Similarly, the 75- $\mathrm{dB}$ chirp level corresponded to the intensity of the F2 transition in $92-\mathrm{dB}$ speech, and also to the intensity level of the entire $75-\mathrm{dB}$ speech stimulus. The 92-dB chirps compared only to the same intensity level of the entire speech pattern.

Chirp discrimination functions are illustrated in Figure 4. At $58 \mathrm{~dB}$, two discrimination peaks were present in locations on the continuum similar to

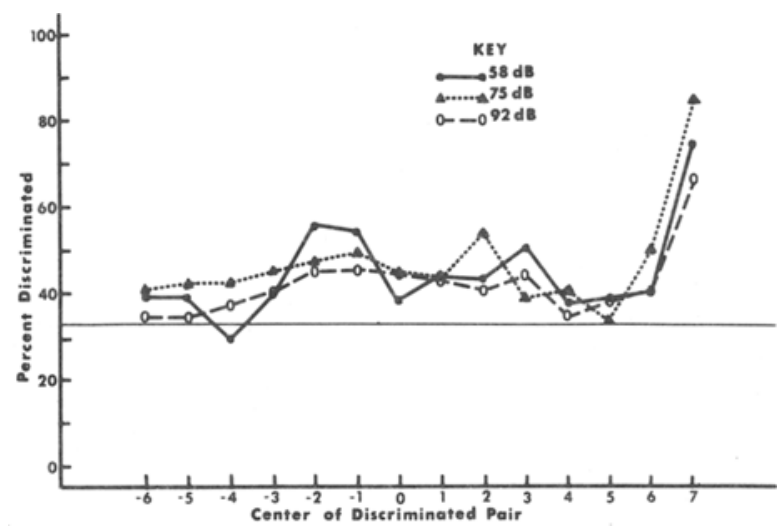

Figure 4. Pooled discrimination functions for chirps at 58, 75 , and $92 \mathrm{~dB}$. 
those of speech peaks. At all intensity levels, a very high level of discrimination occurred at discrimination pair 7 (the comparison of Stimuli 6 and 8) at the high-frequency end of the continuum. A repeated measures analysis of variance, like those described for speech discriminations, was performed using only chirp discrimination pairs -7 to 6 . Data from discrimination pair 7 was omitted from the analysis, because its extremely high discrimination across all intensity levels would increase variability and make analysis of the two other chirp peaks corresponding to speech peaks difficult. The analysis indicated that there were significant differences between the overall discrimination of different discrimination pairs $(\mathrm{F}=2.47, \mathrm{df}=12 / 180, \mathrm{p}<.005)$. Tukey tests for specific contrasts (with $\mathrm{p}<.05$ ) indicated that at $58 \mathrm{~dB}$ there were two peaks-consisting of pairs $-2,-1$, and 3 . the lower frequency peak was significantly higher than troughs at both ends and the trough between the peaks. The higher frequency peak was significantly higher than only the trough at the lower frequency end of the continuum. At $75 \mathrm{~dB}$, peaks occurred at pair -1 and pair 2 , and another small peak at pair 6 . These peaks were significantly higher than only one, two, and one other pairs on the continuum, respectively. There were no significant differences between any discrimination pairs at $92 \mathrm{~dB}$.

Discrimination functions of all six subjects in b-d-g, p-t-k, and chirp conditions were compared at different intensity levels by a series of Stimulus Condition by Discrimination Pair repeated measures analyses of variance, with both conditions and discrimination pairs as repeated factors. In these analyses, 75-dB b-d-g and p-t-k discriminations were compared with 58-dB chirps in one ANOVA and with 75-dB chirps in another ANOVA. Discriminations of $92 \mathrm{~dB}$ b-d-g and p-t-k were similarly compared with 75-dB chirps and with 92-dB chirps. Discrimination pair 7 was eliminated from the analyses in all conditions because of the extremely high discriminability of that pair in the chirp condition. There was no significant overall difference in discrimination between $58-\mathrm{dB}$ chirps and $75-\mathrm{dB}$ b-d-g and $\mathrm{p}$-t-k discriminations; a significant overall difference was observed between discrimination pairs $(\mathrm{F}=7.31$, df $=12 / 180, \mathrm{p}<.001)$, and there was a significant interaction in the location of discrimination peaks between stimulus conditions $(\mathrm{F}=2.68$, $\mathrm{df}=$ $24 / 180, p<.001)$. Tukey tests of specific contrasts (with $\mathrm{p}<.05$ ) indicated that there were no significant differences between the heights of b-d-g and p-t-k discrimination peaks, or between the heights of $p-t-k$ and chirp peaks, or between the size of the b-d peak and corresponding chirp peak, but there was a significant difference in peak height between the highest point of the d-g peak and the higher frequency chirp peak. Figure 5 shows the 75-dB speech and 58-dB chirp discrimination functions. It can be seen that $\mathrm{p}-\mathrm{t}-\mathrm{k}$ and chirp functions were quite similar in peak size and location, but b-d-g peaks were somewhat higher and occupy lower frequency locations. In the analysis of variance comparing $75-\mathrm{dB}$ discrimination functions of the three sets of stimuli, the only differences from the 58-dB chirp and 75-dB speech comparison were the significant difference between the highest point of the 75-dB b-d peak and all 75-dB chirp discrimination peaks, and the fact that the $\mathrm{d}-\mathrm{g}$ peak at $75 \mathrm{~dB}$ was not significantly higher than the corresponding 75-dB chirp peak. In the analysis of variance comparing 75- $\mathrm{dB}$ chirp discrimination with 92-dB b-d-g and p-t-k discrimination, a significant overall difference between discrimination pairs was observed $(F=2.33, \mathrm{df}=12 / 180, \mathrm{p}<.008)$, but there was no significant difference between the location or height of discrimination peaks over the three conditions. Similarly, when discrimination was compared between chirp, b-d-g, and p-t-k conditions at $92 \mathrm{~dB}$, there was a significant overall difference between discrimination pairs $(F=2.37$, df $=12 / 180$, $\mathrm{p}<.007$ ), but no significant difference between the pattern of discrimination or peak heights over the three conditions.

The lower four rows of Table 2 show the chisquare values comparing obtained chirp discrimination functions with predicted speech discrimination functions at the same intensities of F2. In comparing obtained chirp discrimination functions with predicted speech functions, discrimination pair 7 was omitted for the same reasons it was omitted in the analyses of variance containing chirp data. The poor match between 75-dB b-d-g predicted and 58-dB chirp obtained functions was of the same magnitude and due to the same causes as the poor matches between the 75-dB and 92-dB-plus-noise b-d-g functions. The other chi-square comparisons reveal a relatively good

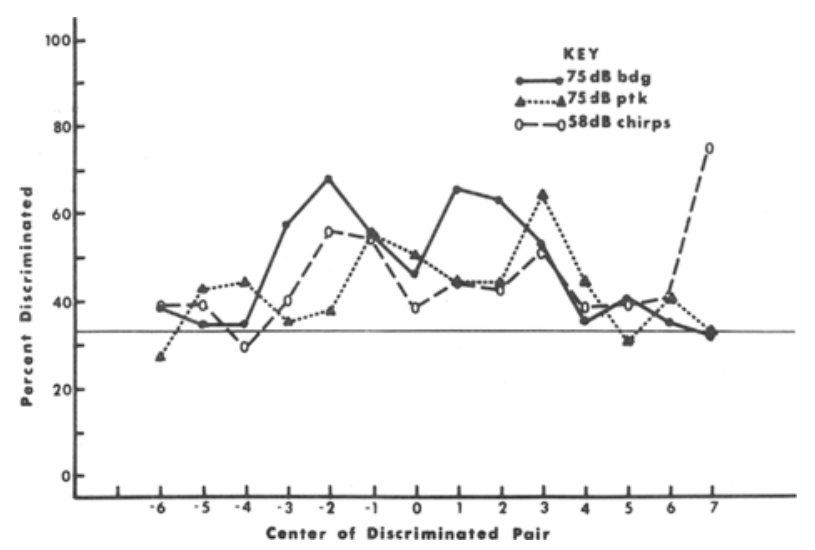

Figure 5. Pooled discrimination functions for 75-dB voiced stops, 75-dB voiceless stops, and 58-dB chirps. 
match between predicted speech and obtained chirp discrimination functions - the values were comparable to those of the more closely matched speech conditions.

The two major observations concerning the chirp discrimination functions are the consistently high discrimination performance at chirp discrimination pair 7, and the presence of two 58-dB chirp discrimination peaks of the same height and at about the same locations as the 75-dB p-t-k boundary peaks.

The high level of discrimination at the highfrequency end of the chirp continuum was unexpected; nothing analogous in the speech discrimination functions was observed at that location. The likely explanation is that the highest frequency stimulus (8) was synthesized slightly differently than the other 15 stimuli. The F2 transition of Stimulus 8 was constructed such that the transition was not so continuous as the other 15 . The F2 transitions of Stimuli -7 to 7 never changed more than approximately $77 \mathrm{cps}$ in $5 \mathrm{msec}$ (one frequency "step"). Between the two initial 5-msec intervals in Stimulus 8, however, the frequency changed by $155 \mathrm{~Hz}$ (two frequency steps). It is interesting that a highly discriminable acoustic cue in isolation was not effective in speech context.

In comparing discrimination performance between b-d-g, p-t-k, and chirp conditions, it is evident that, in several respects, $p$-t-k and chirp discrimination functions were more similar to one another than were b-d-g and p-t-k or b-d-g and chirp functions. The discrimination peak heights of 58-dB chirps and 75-dB $\mathrm{p}-\mathrm{t}-\mathrm{k}$ functions were very similar, as were their locations along the continuum. The 75-dB b-d-g discrimination peaks, however, were somewhat higher, and the peaks were located at a lower frequency than the p-t-k and chirp peaks. The memory demands of the oddity discrimination procedure could have favored discrimination of those stimuli- $\mathrm{b}-\mathrm{d}-\mathrm{g}$ and $\mathrm{p}-\mathrm{t}-\mathrm{k}-$ which were classified and stored in phonetic memory, a presumably longer lasting memory system than acoustic memory, where chirps would have been stored. But, since chirp discrimination peaks were somewhat lower than only the b-d-g peaks, and similar in height to the p-t-k peaks, the explanation for their differences cannot be the differences between acoustic and phonetic memories, or backward masking by the steady-state portions of the stimulus on the F2 transition. The differences between b-d-g discrimination and p-t-k and chirp discrimination must be attributable to acoustic differences between them. Chirps and F2 transitions in p-t-k context were unmasked by simultaneously occurring components of the stimulus. F2 transitions in b-d-g context, however, were partially masked by the simultaneously present lower frequency F1 and hence of lower effective intensity.
Typically, previous studies which have compared the perception of stop consonants and isolated second formant components have not reported the intensity level at which stimuli were presented, or have simply reported that stimuli were presented at approximately equal intensities. Consequently, the $\mathrm{F} 2$ transition in isolation was presented at a higher intensity than the F2 transition in speech context. The Mattingly et al. (1971) study, however, which compared discrimination of voiced stops, isolated second formants, and second formant transitions, presented these stimuli such that the isolated $\mathrm{F} 2$ components were equated in intensity, rather than equated in the overall intensity of the various stimuli. Categorical perception was observed with voiced stops, but, with one exception, not with isolated $F 2$ components. The reason for the categorical perception of isolated F2 transitions in the present study but not in the Mattingly et al. study may be traceable to the intensity of the F2 transition. The stimuli employed in the Mattingly et al. (1971) study contained an F2 transition synthesized at an intensity parameter $6 \mathrm{~dB}$ higher than the intensity parameter employed in the synthesis of the stimuli used in the present study. The intensity level at which the Mattingly et al. stimuli were presented was not measured, so the intensities of the F2 components in that study and the F2 transitions in the present study cannot be directly compared. However, the Mattingly et al. stimuli did contain an F2 transition higher in intensity relative to the other components of the stimulus than the stimuli used in the present study. It is possible that the Mattingly et al. F2 transitions were sufficiently low in effective intensity when masked by $F 1$ in voiced stop context to produce categorical perception, but too high in intensity when unmasked by F1 to be perceived reliably in a categorical fashion.

If effective intensity of the F2 transition determines the location of identification boundaries and the location and height of discrimination peaks, the question remains: What actually is the auditory cue underlying the categorical perception observed in these experiments and how does intensity affect it? Furthermore, why should a lower intensity stimulus produce higher discrimination peaks when just the opposite effect would seem more intuitively likely?

\section{GENERAL DISCUSSION}

Apparently phonetic categories are not extracted or computed by the listener in certain specified locations along a frequency continuum from continuous auditory data; if they were, we would not expect to see the changes in location of boundary loci and peaks or the poorer between-category performance in speech conditions at high intensity which were observed in the present study. Instead, it would seem 
that underlying auditory qualities or features are experienced directly and reflect qualitative, not quantitative, perceptual differences.

Several studies of the detection of frequency changes of pure tones have found evidence for two different modes of perceiving pitch transitions. Pollack (1968) found that when the initial frequency of tone transitions was constant, listeners appeared to compare the initial and final pitches perceived in the transition and based their judgments of whether or not the tone changed in pitch on the discriminable difference between initial and final frequencies. However, with a variable initial frequency of the transitions, Pollack found that listeners could respond directly to frequency transitions. Tsumura, Sone, and Nimura (1973) also suggested that there were two mechanisms for auditory detection of frequency transitions. They found that when a sufficiently long steady-state portion of the tone preceded or followed a transition, listeners tended to detect a pitch transition when they could discriminate a difference between the initial and final pitches of the tone. When the tone had sufficiently short steady-state portions preceding or following the transition, the listener tended to detect a transition from the sensation of a gliding pitch. In both studies, which strategy a listener employed depended upon the amount of pitch information available in the stimuli and the task procedure.

The two strategies discussed by Pollack (1968) and Tsumura et al. (1973) suggest two analogous strategies that might apply to the present study. Listeners could discriminate $F 2$ transitions in speech or nonspeech contexts by comparing the initial pitches of the transitions to be discriminated; the final frequency of F2 transitions was constant in all stimuli. Discrimination functions resulting from a pitch comparison strategy would be expected to be continuous, since a listener could discriminate the constant difference of $155 \mathrm{~Hz}$ in the initial $\mathrm{F} 2$ frequencies being compared approximately equally well along the range of the frequency continuum studied. Listeners could also discriminate $F 2$ transitions by a transitional quality strategy; stimuli could be perceived as having a rising F2 transition, no transitional quality in F2, or a falling F2 transition, and could be discriminated from one another only when their transitional qualities differed. A discrimination function with two peaks and three trough areas would be predicted from a transitional quality model. It may be that when the F2 is heard at a high intensity, there is sufficient information about the initial pitch of the transition available to the listener to make a discrimination judgment based on differences in initial pitch. When the $F 2$ is heard at a low intensity or is masked by noise, perhaps information about initial pitch is insufficient to allow the listener to make a discrimination based on initial pitch differences, and he employs a transitional quality strategy instead. The transitional quality strategy would be expected in the case of normal speech stimuli, which contain transitions of low intensity, especially when simultaneous masking by other formants is considered.

If a transitional quality strategy were employed by a listener, the width of the middle category would be expected to increase with poorer listening conditions, such as noise or low intensity, because presumably it would be more difficult to detect a transition. Several such changes were observed in the present study. The alveolar category width was significantly greater at $75 \mathrm{~dB}$ than at $92 \mathrm{~dB}$ and $92 \mathrm{~dB}$ plus noise. Differences between alveolar category width at $92 \mathrm{~dB}$ and $92 \mathrm{~dB}$ plus noise were not significant, however. Over all intensities, the $t$ category was significantly narrower than the $d$ category (for which perception of F2 transitional quality would be more difficult due to simultaneous masking by F1). As middle categories broaden under poorer listening conditions, one would expect boundary changes to occur on both sides of the middle category. Overall, the bilabialalveolar boundary was of significantly lower frequency at $75 \mathrm{~dB}$ than at $92 \mathrm{~dB}$, but the alveolar-velar boundary was essentially unchanged. Only when noise was added to 92 -dB stimuli did the alveolar-velar boundary move to a significantly higher frequency than it occupied at 75 and $92 \mathrm{~dB}$, but there was no significant change in the location of the bilabial-alveolar boundary between $92 \mathrm{~dB}$ and $92 \mathrm{~dB}$ plus noise. The resistance of the alveolar-velar boundary locus and discrimination peaks to move between 75 and $92 \mathrm{~dB}$ may tend to disconfirm the transitional quality hypothesis, or may indicate that increasing intensity does not affect the detection of rising and falling transitions in the same way (perhaps related to the asymmetry of the envelope of displacement of the basilar membrane).

A problem involved with comparing $b-d-g$ and $p-t-k$ discrimination peak changes between $92 \mathrm{~dB}$ and other intensities is that the peaks were very small or frequently absent at $92 \mathrm{~dB}$ in individual discrimination functions. It may be that much of the perceptual difference between 92-dB and 92-dB-pius-noise stimuli involved a change in listeners' strategy from primarily a pitch-comparison mode at $92 \mathrm{~dB}$ to a transitional quality mode at $92 \mathrm{~dB}$ plus noise.

The pitch comparison and transitional quality strategy model suggested above can account for many of the results observed in the present study, although questions remain concerning others. The model seems to merit further study. Whether the model is valid or not, the categorical-like perception of low-intensity chirps implies an auditory basis for the categorical perception of stops differing in place of articulation. However, it is not clear whether the phenomenon results from general auditory mechanisms 
or from specialized human speech detectors which may be activated by acoustic features characteristic of speech, but not necessarily limited to speech.

\section{REFERENCE NOTES}

1. Fujisaki, H., \& Kawashima, T. On the modes and mechanisms of speech perception. Annual Report of the Engineering Research Institute, University of Tokyo, 1969, 28, 67-73.

2. Fujisaki, H., \& Kawashima, T. Some experiments on speech perception and a model for the perceptual mechanism. Annual Report of the Engineering Research Institute, University of Tokyo, 1970, 29. 207-214.

3. Pisoni, D. B. The role of auditory short-term memory in vowel perception. Status Report on Speech Research, Haskins Laboratories, 1973, SR-34, 89-118.

4. Sachs, R. M. Vowel identification and discrimination in isolation vs. word context. Quarterly Progress Report, Research Laboratory of Electronics, MIT, Cambridge, Massachusetts, 1969, No. 93, 220-229.

\section{REFERENCES}

Abramson, A. S., \& LISker, L. Discriminability along the voicing continuum: Cross-language tests. In Proceedings of the 6th International Congress of Phonetic Sciences (Prague, 1967). Prague: Academia, 1970. Pp. 569-573.

Cutting, J. E., \& Dorman, M. F. Discrimination of intensity differences carried on formant transitions varying in extent and duration. Perception \& Psychophysics, 1976, 20, 101-107.

Cutring, J. E., \& Rosner, B. S. Categories and boundaries in speech and music. Perception \& Psychophysics, 1974, 16, 564-570.

Dorman, M. Discrimination of intensity differences on formant transitions in and out of syllable context. Perception \& Psychophysics, 1974, 16, 84-86.

Eimas, P. D., \& Corbit, J. D. Selective adaptation of linguistic feature detectors. Cognitive Psychology, 1973, 4, 99-109.

Ellrotr, L. Backward masking: Monotic and dichotic conditions. Journal of the Acoustical Society of America, 1962, 34, 1108-1115.

Flanagan, J. L., \& Saslow, M. G. Pitch discrimination for synthetic vowels. Journal of the Acoustical Society of America, $1958,30,435-442$.

Jusczyk, P. W., Rosner, B. S., Cutting, J. E., Foard, C. F., \& Sмiтн, L. B. Categorical perception of nonspeech sounds by 2 -month-old infants. Perception \& Psychophysics, 1977, 21, 50-54.

Kuhl, P. K., \& Miller, J. D. Speech perception by the chinchilla: Voiced-voiceless distinction in alveolar plosive consonants. Science, 1975, 190, 69-72.

LANE, H. L. The motor theory of speech perception: A critical review. Psychological Review, 1965, 72, 275-309.

Liberman, A. M., Harris, K. S., Hoffman, H. S., \& Griffith, B. C. The discrimination of speech sounds within and across phoneme boundaries. Journal of Experimental Psychology, 1957, 54, 358-368.

LISKER, L., \& ABRAMSON, A. S. The voicing dimension: Some experiments in comparative phonetics. In Proceedings of the 6th International Congress of Phonetic Sciences (Prague, 1967). Prague: Academia, 1970. Pp. 563-567.

MASSARo, D. W. A comparison of forward versus backward recognition masking. Journal of Experimental Psychology, $1973,100,434-436$.

MASSARO, D. W. Backward recognition masking. Journal of the Acoustical Society of A merica, 1975, 58, 1059-1065.

Mattingly, I. G., Liberman, A. M., Syrdal, A. K., \&
HALWES, T. Discrimination in speech and nonspeech modes. Cognitive Psychology, 1971, 2, 131-157.

McGovern, K., \& Strange. W. The perception of $/ \mathbf{r} /$ and $/ 1 /$ in syllable-initial and syllable-final position. Perception \& Psychophysics, 1977, 21, 162-170.

MilleR, G. A. Language and communication. New York: McGraw-Hill, 1951.

Miller, G. A. The magical number seven plus or minus two: Some limits on our capacity for processing information. Psychological Review, 1956, 63, 81-97.

Miller, J. D.. Wier, C. C., Pastore, R. E., Kelly, W. J., \& Dooling, R. J. Discrimination and labeling of noise-buzz sequences with varying noise-lead times: An example of categorical perception. Journal of the Acoustical Society of America, 1976, 60, 410-417.

Miller, J. L., \& Eimas, P. D. Studies on the perception of place and manner of articulation: A comparison of the labialalveolar and nasal-stop distinctions. Journal of the Acoustical Society of America, 1977, 61, 835-845.

MiYawaki, K., Strange, W., Verbrugge, R., Liberman, A. M., Jenkins, J. J., \& Fujimura, O. An effect of linguistic experience: The discrimination of $[\mathrm{r}]$ and $[\mathrm{I}]$ by native speakers of Japanese and English. Perception \& Psychophysics, 1975, 18. 331-340.

Pastore, R. E., Ahroon, W. A., Puleo, J. S., Crimmins, D. B., Golowner. L., \& Berger, R. S. Processing interaction between two dimensions of nonphonetic auditory signals. Journal of Experimental Psychology: Human Perception and Performance, 1976, 2, 267-276.

PIsoni, D. B. On the nature of categorical perception of speech sounds. Doctoral dissertation, University of Michigan, 1971. (Published as Supplement to Status Report on Speech Research, Haskins Laboratories, 1971.)

PIsoni, D. B. Auditory and phonetic memory codes in the discrimination of consonants and vowels. Perception \& Psychophysics, 1973, 13. 253-260.

Pisonr, D. B. Identification and discrimination of the relative onset time of two component tones: Implications for voicing perception in stops. Journal of the Acoustical Society of America, 1977, 61, 1352-1361.

Pollack, 1. The information in elementary auditory displays. Journal of the Acoustical Society of A merica, 1952, 24, 745-749.

PoLLACK, 1. Detection of rate of change of auditory frequency. Journal of Experimental Psychology, 1968, 77, 535-541.

POPPER, R. D. Pair discrimination for a continuum of synthetic voiced stops with and without first and third formants. Journal of Psycholinguistic Research, 1972, 1, 205-219.

RAND, T. C. Dichotic release from masking for speech. Journal of the Acoustical Society of America, 1974, 55, 678-680.

STEVENs, K. N. On the relations between speech movements and speech perception. Zeitschrift für Phonetik. Sprachwissenschaft und Kommunikationsforschung, 1968, 213, 102-106.

Stevens, K. N., \& K KaTT, D. H. Role of formant transitions in the voiced-voiceless distinction for stops. Journal of the Acoustical Society of America, 1974, 55, 653-659.

Studdert-Kennedy, M., Shankweiler, D. P., \& Schulman, S. Opposed effects of a delayed channel on perception of dichotically and monotically presented CV syllables. Journal of the Acoustical Society of America, 1970, 48, 599-602.

Tsumura, T., Sone, T., \& Nimura, T. Auditory detection of frequency transition. Joumal of the Acoustical Society of America, 1973, 53, 17-25.

WILSON, R. H., \& CARHART, R. Forward and backward masking: Interactions and additivity. Joumal of the Acoustical Society of America, 1971, 49, 1254-1263.

(Received for publication May 24, 1977; revision accepted February 26, 1978. ) 